\section{EPV248/\#405 A THEMATIC ANALYSIS OF KNOWLEDGE AND MISINFORMATION AMONG CERVICAL CANCER RADIOTHERAPY PATIENTS AT A TERTIARY HOSPITAL IN SOUTH AFRICA}

${ }^{1} \mathrm{H}$ Simonds*, ${ }^{2} \mathrm{R}$ Williams, ${ }^{2} \mathrm{R}$ Roomaney. 'Stellenbosch University, Radiation Oncology, Cape Town, South Africa; '2Stellenbosch University, Department of Psychology, Cape Town, South Africa

\subsection{6/ijgc-2021-IGCS.319}

Objectives The high prevalence and burden of cervical cancer in developing countries has spurred on much research into preventing and screening for the disease. However, little research has focussed on the experience of living with the disease and undergoing treatment for it in South Africa. In this study we aim to report on the knowledge, misinformation, stigma and disclosure hesitancy among women receiving curative treatment for cervical cancer at a tertiary hospital in South Africa

Methods Inclusion criteria included being between the ages of 18 and 50 years and having undergone curative treatment for invasive cervical cancer, which resolved no more than 18 months prior to interviewing them. We conducted semi-structured interviews. Interviews were audio-recorded, transcribed, and analysed using thematic analysis.

Results Fifteen women between the ages of 28 to 49 years old participated in the study. We describe these 15 participants' knowledge and understanding of cervical cancer, their experience of misinformation and stigma and a hesitancy to disclose their illness to others. Participants reported that they knew very little about cervical cancer, its causes, symptoms, diagnosis, and treatment. Women reported that they encountered misinformation and that in some cases this led to delays in diagnosis. One prominent negative perception that they encountered was the association of cervical cancer with promiscuity. Overall, participants seemed hesitant to disclose their diagnosis with others.

Conclusions We highlight the central role that communication can play in increasing knowledge, reducing stigma and misinformation, and facilitating disclosure among women with cervical cancer. We include recommendations for healthcare practitioners and researchers.

\section{EPV249/\#482 RACIAL DISPARITIES IN THE COMPLETION OF THE HPV VACCINATION SERIES IN WOMEN IN THE UNITED STATES - A 9-YEAR STUDY}

${ }^{1} \mathrm{C}$-I Liao, ${ }^{2} \mathrm{~A}$ Milki, ${ }^{3} \mathrm{D}$ Wong, ${ }^{4} \mathrm{E}$ Thayer, ${ }^{5} \mathrm{~A}$ Moon, ${ }^{6} \mathrm{~A}$ Chan, ${ }^{3} \mathrm{~A}$ Francoeur, ${ }^{6} \mathrm{~J}$ Chan. ${ }^{1}$ Kaohsiung Veterans General Hospital, Obstetrics and Gynecology, Kaohsiung City, Taiwan; ${ }^{2}$ The George Washington University, School of Medicine and Health Sciences, Washington, USA; ${ }^{3}$ University of California Los Angeles, Obstetrics and Gynecology, Los Angeles, USA; ${ }^{4}$ University of Massachusetts Medical School, Obstetrics and Gynecology, Worcester, USA; ${ }^{5}$ Stanford University School of Medicine, Obstetrics and Gynecology, Division of Gynecologic Oncology, Stanford, USA; ${ }^{6}$ California Pacific Medical Center, Obstetrics and Gynecology, San Francisco, USA

\subsection{6/ijgc-2021-IGCS.320}

Objectives To identify disparities and trends in HPV vaccination status and trends across races.

Methods HPV vaccination rates were evaluated using the Behavioral Risk Factor Surveillance System (BRFSS). Joinpoint regression program 4.8.0.1 was used to calculate the trend (average annual percent change, AAPC).
Results In all patients, there was an increase in 3-dose vaccination from 2008 to $2016 \quad(3.6 \%$ annual percent change (APC), $\mathrm{p}=0.042$ ). There was also an increase in APC of 19.4\% between 2008 and $2010(\mathrm{p}=0.046)$. When examining only those between 18-24 years of age, there was an APC of $26 \%(p=0.037)$. From 2010 to 2016 , there was no significant APC in all age groups assessed. In 2008, 27.78\% of Blacks, $25 \%$ of Hispanics, $48.78 \%$ of Whites, and $42.86 \%$ of Asians had received 3 vaccine doses. In 18 to 24 year old Black patients, there was an APC of $77.3 \%$ from 2008 to $2010(p=0.027)$, followed by a $-7.4 \%$ APC $(p=0.053)$ from 2010 to 2016. Hispanic patients also showed a significant increase in vaccine completion; a 43.9\% APC took place from 2008 to $2010 \quad(\mathrm{p}=0.040)$ among 18-24-year-old patients, and a $39.7 \%$ in APC took place in the total Hispanic population $(\mathrm{p}=0.033)$.

Conclusions The majority of the increase in 3-dose vaccination that took place across all races from 2008 to 2016 took place in the first 3 years of that time period. Black and Hispanic patients were least likely to have 3 doses of the HPV vaccine in 2008, but experienced the greatest increases in rates of vaccine completion between 2008 and 2010.

\section{EPV250/\#600 ASSOCIATIONS OF HEALTHCARE AFFORDABILITY WITH GUIDELINE-ADHERENT SURGERY AMONG WHITE, BLACK, AND HISPANIC OVARIAN CANCER PATIENTS: A U.S NATIONAL CANCER DATABASE ANALYSIS}

${ }^{1,2} \mathrm{O}$ Ohamadike*, ${ }^{2} \mathrm{~L}$ Wilson, ${ }^{2,3} \mathrm{~A}$ Gupta, ${ }^{2} \mathrm{~T}$ Akinyemiju. ${ }^{1}$ Duke University School of Medicine, Department of Obstetrics and Gynecology, Durham, USA; ${ }^{2}$ Duke University School of Medicine, Department of Population Health Sciences, Durham, USA; ${ }^{3}$ Duke University, Trinity College of Arts and Sciences, Durham, USA

\subsection{6/ijgc-2021-IGCS.321}

Objectives Black and Hispanic ovarian cancer (OC) patients are less likely to receive guideline-adherent and timely cancer care. We aimed to evaluate the associations between healthcare affordability (HA) and receipt of high-quality surgery and time to surgery among OC patients by race.

Methods Data from the 2016 NCDB on Non-Hispanic White (NHW), Non-Hispanic Black (NHB), and Hispanic OC patients diagnosed in 2004-2016 was analyzed. Measures of HA included area-level income and insurance status. Multinomial logistic regression was used to estimate the odds of receiving high-quality surgery compared with lowquality or no surgery. Multivariable linear regression was used to analyze differences in time, in days, from diagnosis to surgery.

Results The cohort included 113,702 patients: $86 \%$ NHW, $8 \%$ NHB and $6 \%$ Hispanic. Compared to private insurance, uninsured and Medicaid patients were more likely to receive no surgery (uninsured: aOR 2.55; 95\% CI 2.28-2.85 and Medicaid: aOR 2.05; 95\% CI 1.86-2.26). Lower income patients were more likely to receive low-quality surgery (aOR 1.20; 95\% CI 1.13-1.27) or no surgery (aOR 1.42; 95\% CI 1.311.53) relative to higher income patients. These associations were strongest among uninsured Hispanic and lowest income NHB patients. Relative to private insurance, Medicaid patients were more likely to have longer time to surgery (b 6.09; $95 \%$ CI 4.17-8.02). This association was strongest among NHB and Hispanic Medicaid patients. 